\title{
TUMOR DE CELULAS DE LA GRANULOSA TEMPRANO: DIFERENCIAS CON LOS CASOS JUVENIL Y DEL ADULTO. REVISIÓN DE LA BIBLIOGRAFÍA. COMENTARIO.
}

\author{
José Pereda-Garay ${ }^{1}$
}

\begin{abstract}
RESUMEN
Se revisa las características clínicas y el cariotipo del tumor de Células de la Granulosa, del feto y el recién nacido, para compararlos con casos de otras edades, descritas en la literatura. Se identifica un grupo de tumores del testículo con diferencias, tanto clínicas como en su cariotipo, por lo que se le debe considerar como un cuadro clínico aparte, que merece ser estudiado.
\end{abstract}

Palabras clave: Tumor de células de la granulosa (tCG); Tumor de células de la granulosa del adulto (tCGA);Tumor de células de la granulosa juvenil (tCG) (Fuente: DeCS BIREME).

\section{GRANULOSA CELL TUMOUR EARLY: DIFFERENCES WITH JUVENILE AND ADULT CASES. LITERATURE REVIEW. COMMENT.}

\begin{abstract}
Clinical picture and karyotype of perinatal cases of Granulosa Cell tumor are reviewed comparing them with cases of other age groups described in the literature. A group of Granulosa Cell Tumor in the testis of newborns presents clinical differences, as in their karyotype, and should be considered with merits to be studied.
\end{abstract}

Key words: Granulosa cell tumor (tCG);Granulosa cell tumor adult type (tCGA); Granulosa cell tumor juvenile type (tCGJ) (Source:MeSH NLM).

\section{INTRODUCCIÓN}

La observación de dos casos de tCG, durante la revisión de rutina de necropsias perinatales del INMP, fue motivo para estudiar las características de esta neoplasia en el feto y el recién nacido, con el objetivo de ver si hay diferencias con los casos que se describen en otras edades; para lo cual se revisó la literatura a nuestro alcance. Las historias clínicas de los casos mencionados, no son relevantes; en ambos la causa de muerte estuvo relacionada con la evolución del parto. El diagnóstico de la neoplasia fue un hallazgo en el examen microscópico de las láminas de las necropsias. En ambos casos el tumor fue ovárico.

Se ha propuesto, para identificar a los tsCG del grupo de fetos y recién nacidos, la denominación de tumor de Células la Granulosa Temprano. Podría llamarse perinatal, pero se ha extendido hasta el primer año de vida ya que algunos casos serán llevados a consulta después del primer mes de vida; el extremo superior es arbitrario. Hay casos en los que el diagnóstico del tumor, ha sido pre-natal ${ }^{1-3}$. En consecuencia, los casos de este grupo evidentemente son congénitos, pero no son, por la edad, ni juveniles ni del adulto. En todo caso el término propuesto está de acuerdo con la nomenclatura de la clasificación de Scully. Al revisar la literatura de este tumor, las diferencias que se encuentran están en el cariotipo y el cuadro clínico que resulta. Se comentará esas diferencias, entre grupos portadores de esta neoplasia.
El tumor de Células de la Granulosa (tCG) es una lesión gonadal. Puede llamar la atención que órganos con características macro y microscópicas diferentes, presenten el mismo tumor. En comparación, si examinamos la morfología de órganos de los otros sistemas de individuos de sexos diferentes, no podemos, por el examen morfológico de rutina, especificar a qué sexo pertenece la muestra. Por el contrario, el ovario y el testículo, tienen características anatómicas tan diferentes, que permiten el diagnóstico diferencial del sexo de origen. La explicación, está en su origen embriológico común.

En este contexto, el sistema genital tiene otra peculiaridad; a diferencia de los otros sistemas de la economía que sirven al individuo; el sistema genital está principalmente al servicio de la especie. Con participación de ambas gónadas: el óvulo del ovario unido a uno de los espermatozoides del testículo, contribuyen con 23 cromosomas cada uno, a formar el huevo o cigote, con 46 cromosomas, punto de partida de un nuevo ser. Este es su objetivo. Esto es lo biológicamente normal.

Las gónadas se forman a partir de la cresta genital; cuyo tejido conectivo al evolucionar constituirá su estroma, con características estructurales y funcionales propias. Uno de estos tejidos se diferenciará, en el ovario, para formar las células de la granulosa, en las que se inicia la neoplasia motivo de este trabajo.

Profesor Principal, Departamento de Patología. Universidad Peruana Cayetano Heredia. Patólogo Consultor. Instituto Nacional Materno Perinatal.

Recibido: 16-01-16 Aprobado: 30-06-16 


\section{COMENTARIO}

Las características clínicas del tCG y su manejo han sido bien estudiados en pacientes adultos y juveniles; $y$ la literatura al respecto es abundante y conocida, por lo que no se la citará; pero en relación al grupo de casos perinatales se encontró pocos estudios específicos. No se ha encontrado referencias en las que se describa el cariotipo de tumores del ovario en la etapa perinatal. Se va a presentar diferencias encontradas en el complemento cromosómico y el cuadro clínico que resulta y comentar las investigaciones de patología molecular que se han descrito en la literatura revisada, para incentivar su aplicación en el grupo que estamos estudiando.

\section{Anomalías cromosómicas en el tCG}

El estudio de las anomalías cromosómicas de los tCG presenta varios problemas:

a) En la mayoría de casos publicados, casos individuales o grupos, no se describe el cariotipo; en estos no se puede concluir si ha habido o no cromosomopatías;

b) en los trabajos que describen cariotipos hay también casos individuales o grupos de casos, pero en varios de estos últimos se describe casos de tsCG junto a otros tumores del estroma de los cordones sexuales con la misma alteración cromosómica; en estos casos solamente se ha tabulado a los tsCG. Si la serie describe cariotipo en ovario y testículo, los casos se tabularon en las tablas de las gónadas respectivas;

c) cuando se describe una serie con técnicas diversas se puede encontrar diferencias dentro del mismo grupo. Por ejemplo, en una serie de 20 casos de tCG se encuentra que 18 de los cariotipos son diploides con la técnica de citometría del ADN; pero en 6 de 16 de ellos $(37 \%)$ usando la técnica FISH se encuentra aberraciones cromosómicas ${ }^{4}$;

d) se ha encontrado casos de tsCG con cariotipo normal.

Por estas razones, anteriormente, se ha considerado discutible el papel de los cromosomas en la patogenia de este tumor.

Teniendo en cuenta lo señalado en el párrafo anterior, para los efectos del presente trabajo, se decidió en primer lugar clasificar las anomalías cromosómicas descritas en la literatura, según la gónada afectada, lo que no se ha hecho anteriormente. El resultado son las tablas, 1 para los tsCG del ovario y la tabla 2 para los tsCG del testículo. Se compara los cariotipos del grupo perinatal de tumores del testículo con los disponibles en la literatura de dichas neoplasias en el ovario, debido a que no se ha encontrado casos de tsCG del ovario, con cariotipo descrito en el grupo perinatal.

En la tabla 1 se observa que las anomalías cromosómicas de los tumores del ovario se encuentran en los autosomas.
Hay algunos casos con trisomía o monosomía $X$; pero en casos de cariotipos complejos dentro de los que hay pérdida y ganancia en diferentes pares de autosomas y los autores no les dan importancia patogénica.

La anomalía que se considera más frecuente en tCGJ es la trisomía del par $12^{5,6}$ pero en varias series se encuentra diversas anomalías en los casos que presentan; además la trisomía 12 no es exclusiva de este grupo; también se le encuentra en tumores del grupo fibroma-tecoma y algunos casos de células de Sertoli ${ }^{7-11}$. Se debe mencionar que Persons $^{12}$ no encuentra trisomía 12 en ninguno de los 24 casos de tsCG de su serie; curiosamente uno de sus casos tiene tetrasomía 12.

En los tsCGA las alteraciones que se consideran relevantes son la monosomía del par 22, algunas veces asociado a trisomía del par 12 y del par $14^{13-16}$. La monosomía 22 se ha usado como ayuda para identificar la naturaleza de un tumor de la granulosa recurrente, en ausencia de las láminas del tumor primario, 21 años después de la ooforectomía ${ }^{17}$; y para diagnosticar un tCG metastásico en pulmón, 20 años después de la extirpación del tumor de ovario ${ }^{18}$

Bouffe ${ }^{19}$ describe un caso de tCG del ovario, con cariotipo normal, 46,XX.

En la tabla 2, los tsCG del testículo se han separado en 3 grupos. El primero corresponde a casos con cariotipo normal ${ }^{20-23}$; en el segundo se incluye ts $C G$ que presentan alteraciones en autosomas, semejantes a los tsCG del ovario ${ }^{24-26}$, en ninguno de estos grupos se describe malformaciones anatómicas, salvo el caso de Koss, pero en éste caso se trata de un aborto por triploidía en el que normalmente se espera diferentes anomalías.

El último grupo de tsCG del testículo (ver tabla 2) se caracteriza por alteraciones del cromosoma Y; éstas son tanto numéricas como estructurales ${ }^{27-33}$. Entre las estructurales hay casos con isocromosoma $\mathrm{Y}$, o cromosoma $Y$ en anillo. Las alteraciones numéricas consisten en mosaicismo 46,XY/45,X. Cuando se encuentra cariotipos con $45, X$ de inmediato se piensa en la monosomía $X$ de la mujer, que presenta un cuadro clínico característico en pacientes de sexo femenino, $46 \mathrm{XX}$, que han perdido un cromosoma $X$, son $46, X X / 45 X,-X$ o simplemente $45, X,-X$ (monosomía $X$, síndrome de Turner). Se propone que en los tsCG del testículo, con cariotipo 46,XY/45,X la alteración numérica se debe a que hay líneas celulares que han perdido el cromosoma Y; son $\mathbf{4 6 , X Y / 4 5 , X , - Y , ~ l o ~ q u e ~ p r o b a b l e m e n t e ~}$ contribuye a la presencia de malformaciones de los genitales. Este laboratorio no ha tenido facilidades para estudiar este problema, pero es interesante señalar que en el trabajo de Schofield y Fletcher, en un grupo de 7 casos, para evaluar el empleo de la técnica de FISH en el estudio de la frecuencia de trisomía 12 en el tCG; uno de sus casos es un tumor de la granulosa del testículo; al aplicar la sonda para testículo, no se encuentra el cromosoma $Y$ en 
los núcleos que examinan. Concluyen que ello se debe a que probablemente el cromosoma $Y$ de dichos núcleos ha perdido el segmento que da la reacción de positividad al $\mathrm{FISH}$, lo que contribuye al desarrollo de esta neoplasia. En ese caso se puede interpretar como que no hay cromosoma Y o que el segmento perdido no causa malformaciones de los órganos genitales. Recientemente, Forsberg y col. han descrito casos de varones con pérdida del cromosoma $Y$, en sangre periférica, en los que se observa un mayor riesgo de cáncer y menor sobrevivencia ${ }^{34}$. Cariotipos $46, X Y / 45, X$ se encuentran también en pacientes con Disgenesia Gonadal. En ninguna de esas series se menciona presencia de tsCG ${ }^{35-37}$.

\section{Aspectos Moleculares en la Oncogénesis del TCG}

El desarrollo de una neoplasia comprende una serie de procesos: desde la iniciación, hasta su diseminación y metástasis. La evolución de las diferentes etapas se realiza, en los tsCG, con la participación de proteínas como la inhibina, la activina, los SMADS (factores de transducción) y factores de transcripción. Cada una de estas proteínas está codificada por genes localizados en diferentes cromosomas. Algunos de dichos genes son oncogenes; otros son oncosupresores.

En el ovario, hay una proteína nuclear: FOXL2 (locus: cromosoma 3 q22.3); que se considera el marcador ovárico más temprano, interviene en la diferenciación del folículo y su desarrollo. No se le encuentra en el testículo. FOXL2 actúa como un factor de transcripción y sus objetivos son, diferentes genes que intervienen en la proliferación, apoptosis y diferenciación celular y genes que participan en la producción de estrógenos. La interacción entre esos genes podría intervenir en el desarrollo del tCG ${ }^{38-41}$.

Shah y col ${ }^{42}$ identificaron una mutación en el gen de FOXL2, en el codón 134; en cuatro casos de tsCG del Adulto que estudiaron. Lo que se ha confirmado en varios trabajos. Estudios posteriores mostraron que la mutación está presente en $97 \%$ de casos de tCGA pero solamente en 1 de 10 (10\%) de los casos del tCGJ. Varios autores han confirmado estos estudios ${ }^{43-46}$.

Llama la atención la diferente frecuencia de la presentación de FOXL2 mutante en el tCGA y el tCGJ. Se sabe, ahora, que estas variedades están constituidas por líneas celulares diferentes; la llamada KGN en la variedad del adulto y la COV434 en la juvenil. La mutación de FOXL2 está marcadamente expresada en células KGN, pero pobremente expresada en las células de la línea COV434.

Si FOXL2 es importante para el desarrollo del tCG en el ovario; se plantea un problema en el caso de los tsCG del testículo, ya que se ha mencionado que FOXL2 no se encuentra en el testículo normal. El factor de transcripción, equivalente, para el testículo es SOX9. En un grupo de tsCG del testículo ${ }^{47}$ han encontrado, en las células tumorales, al gen FOXL2 mutante, localizado en el núcleo, lo que sugiere que tenía actividad biológica; por el contrario SOX9, gen que normalmente se encuentra en el núcleo de las células testiculares, estaba secuestrado en el citoplasma o sub-expresado en el núcleo.

\section{CONCLUSIÓN}

El interés de la presente revisión ha sido estudiar el tumor de células de la granulosa en el feto y el recién nacido y su diferencia con casos de otras edades, habiéndose encontrado, en este grupo, características peculiares. En la literatura revisada solamente se ha encontrado el caso de Bouffet con cariotipo en una infante de 16 meses por esta razón la tabla de tsCG del ovario solamente comprende casos descritos en pacientes de sexo femenino, por encima de la etapa perinatal, en los que tampoco se describe malformaciones genitales. Son casos que se presentan de la infancia a la edad adulta; muchas veces son llevados a consulta por signos de feminización precoz.

En el grupo de los varones se ha identificado ts CG con cariotipo normal y otros con anomalías autosómicas, la evolución clínica de estos casos es similar a la de pacientes con tCG en el ovario.

El último grupo de tsCG del testículo, identificado, presenta un síndrome clínico diferente, que se caracteriza por:

a. Fenotipo masculino o con ambigüedad genital,

b. Presencia de tumor de células de la granulosa, congénito, en el testículo,

c. Anomalías estructurales y/o numéricas del cromosoma $Y$,

d. Malformaciones del aparato genital consistentes en genitales externos ambiguos; en algunos casos con presencia de útero, generalmente unicorne, que se continúa con trompa uterina, que está en relación con una gónada que puede ser por un cordón fibroso formado por estroma ovárico.

Es evidente que las malformaciones descritas están relacionadas a las anomalías del cromosoma $\mathrm{Y}$, que las caracteriza; cuya consecuencia, una importante disminución en la dotación de sus genes, por el menor número de cromosomas $\mathrm{Y}$, se traduciría en una disminución en el número y capacidad funcional de las células de Leydig, cuyos productos la testosterona y su derivado la dihidrotestosterona, intervienen en la formación de los conductos y los genitales externos del varón; y de las células de Sertoli que participan en la producción de la hormona anti-mulleriana. El defecto también podría estar en otras enzimas como la 5 a-reductasa, como se describe en el síndrome de Hipospadias Perineo-escrotal Pseudovaginal por Deficiencia de la $5 \alpha$-Reductasa 2; u otras enzimas con actividad parecida. 
Tabla 1. Cariotipos en tumor de células de la granulosa en ovario

\begin{tabular}{|c|c|c|c|}
\hline Autores & Cariotipo & $\begin{array}{l}\text { Genitales } \\
\text { anómalos }\end{array}$ & Edad \\
\hline \multirow[t]{2}{*}{$\begin{array}{l}\text { Schofield y } \\
\text { Fletcher }\end{array}$} & $47, X X,+12 \quad 2 / 3$ casos tCGJ & NO & - \\
\hline & $47, \mathrm{XX},+12 \quad$ (tCG metastásico) & NO & - \\
\hline \multirow{5}{*}{$\begin{array}{l}\text { Halperin y } \\
\text { col }\end{array}$} & $47, X X,+12$ en $10 / 17$ tCGA & NO & - \\
\hline & 49. XXX, $+12,+17$ en 1 caso & $\mathrm{NO}$ & - \\
\hline & $48, X X X,+12$ & NO & - \\
\hline & en 2 casos & NO & - \\
\hline & $47, X X,+12$ en $2 / 2$ tCGJ & NO & - \\
\hline Shashi & $47, \mathrm{XX},+12$ en $1 / 2$ casos & $\mathrm{NO}$ & - \\
\hline Gaffey & $47, X X,+12$ & NO & - \\
\hline $\begin{array}{l}\text { Rodríguez E } \\
\text { y col }\end{array}$ & 46,XX/49-115 cariotipo complejo & NO & 11 años \\
\hline Teyssier & $\begin{array}{l}45, X / 47, X X,+1 / 46, X X \\
-1 p / 46, X X,-1 q / 46, X X,-6 q\end{array}$ & NO & - \\
\hline Leung & $46, X X+12,-22, t(3 ; 9: 21)$ & $\mathrm{NO}$ & 62 años \\
\hline Dhillon & $46, X X,+14,-22$ & NO & - \\
\hline Gorski & $47, X X,+14,-22$ & NO & 56 años \\
\hline Namiq & $46, X X,+14,-22$ & NO & - \\
\hline $\begin{array}{l}\text { Van den } \\
\text { Berhge }\end{array}$ & $46, X X,+14,-22$ & NO & 59 años \\
\hline Caughron & $45, X X,-22$ & NO & $\begin{array}{l}\text { recurr.21 } \\
\text { dps }\end{array}$ \\
\hline $\begin{array}{l}\text { Lindgren } \vee y \\
\text { col (caso 1) }\end{array}$ & $46, X X,+14,-22 / 46, X X \quad$ tCGA & NO & 41 años \\
\hline $\begin{array}{l}\text { Lindgren V y } \\
\text { col (caso 2) }\end{array}$ & $\begin{array}{l}42, X X,+12,+9,-5,-7,-13,-15,-16,- \\
22 / 46, X X\end{array}$ & NO & 29 años \\
\hline $\begin{array}{l}\text { Fletcher } \\
\text { (caso 8) }\end{array}$ & $47, X X,+12$ & NO & 7 años \\
\hline $\begin{array}{l}\text { Fletcher } \\
\text { (caso 4) }\end{array}$ & $\begin{array}{l}50, X X,,+X,+7 x,+8,+9,+12,-22 \\
\text { tCGA }\end{array}$ & NO & 45 años \\
\hline $\begin{array}{l}\text { Bouffet } E \\
\text { y col }\end{array}$ & 46, XX en 1/3 tCGJ & NO & $\begin{array}{c}16 \\
\text { meses }\end{array}$ \\
\hline Schwartz & $\begin{array}{l}47 X X,+12 / 45 X X,- \\
22 / 46 X X, t(3 ; 9 ; 21)\end{array}$ & NO & $\begin{array}{c}18 \\
\text { meses }\end{array}$ \\
\hline Taruscio & $\begin{array}{l}\text { tcg } 4 \text { casos trisomía }+12+ \\
12-32 \%\end{array}$ & $\mathrm{NO}$ & - \\
\hline
\end{tabular}

Tabla 2. Cariotipos en tumor de células de la granulosa en testículo

\begin{tabular}{|c|c|c|c|}
\hline Autores & Cariotipo & $\begin{array}{l}\text { Genitales } \\
\text { Anómalos }\end{array}$ & Edad \\
\hline Nistal y col & $46, X Y$ & $\mathrm{NO}$ & $\mathrm{RN}$ \\
\hline Alexiev y col & $46, X Y$ & $\mathrm{NO}$ & RN \\
\hline Bulotta & $46, X Y$ & NO & $\mathrm{RN}$ \\
\hline Couture & $46, X Y$ & NO & 3 meses \\
\hline Kos y col & $69, X X Y$ & SI & $\begin{array}{l}\text { Aborto } \\
\text { fetal }\end{array}$ \\
\hline Yu DC y col & $46, X Y,(d e l) 4$ & NO & $\mathrm{RN}$ \\
\hline Schofield \& Fletcher & $47, X Y,+12$ & $\mathrm{NO}$ & 1 mes \\
\hline Young y col (caso 1) & $\begin{array}{c}\text { 45,X/46X, dic } \\
\text { (Yp)/46,XY }\end{array}$ & SI & RN \\
\hline Young y col. (caso 2) & $45, X / 47, X Y Y$ & SI & 3 meses \\
\hline Young y col (caso 3) & $45, \mathrm{X} / 46, \mathrm{X}, \mathrm{rY}$ & SI & $\begin{array}{c}6 \\
\text { semanas }\end{array}$ \\
\hline Raju y col & $45, X / 46, X Y$ & SI & RN \\
\hline Chan JK y col & $45, X / 46, X$, iso(Yq) & SI & 1 mes \\
\hline Tanaka y col & $45, X / 46, X Y$ & SI & 2 meses \\
\hline Plesner y col (caso 1) & $45, X / 46, X, \operatorname{dic}(Y q)$ & SI & Congénito \\
\hline Nitzsche & 45,X/46,X, Y(mar) & SI & RN \\
\hline Pascual J y col & $45, X / 46, X, \operatorname{idic}(Y)$ & SI & RN \\
\hline
\end{tabular}

De 1985 al 2009 se han descrito 9 casos con las características que se han identificado. El objeto de esta presentación es llamar la atención sobre este grupo de tsCG del testículo, para incentivar su búsqueda y estudio en los archivos de laboratorios de Patología Perinatal y en casos clínicos que se presenten en los Servicios de Neonatología, los que deberán incluir cariotipos y la búsqueda de mutaciones génicas. Las respuestas que se obtengan contribuirán a un mejor conocimiento de la biología de esta neoplasia.

Agradecimiento a:

- Carmen Bou-Crick. Librarian Assistant Professor. Louis Calder Memorial Library. School of Medicine University of Miami. Por su valiosa colaboración.

- Elizabeth Ramírez. Coordinadora de Servicios Biblioteca Central. U.P.C.H.

\section{REFERENCIAS BIBLIOGRÁFICAS}

1. Peterson C, Skoog S. Prenatal diagnosis of juvenile granulosa cell tumor of the testis. J Pediatr Urol 2008; 4:472-4

2. Crump WD Juvenile granulosa cell (Sex Cord-Stromal) tumor of fetal testis. J.Urol 1983; 129(5): 1057-8

3. Lawrence WD, Young RH, Scully RE Juvenile granulosa cell tumor of the infantile testis. A report of 14 cases Am J Surg Pathol 9(2): 87-94;

4. Mayr D ${ }^{1}$, Kaltz-Wittmer C, Arbogast S, Amann G, Aust DE, Diebold J. Characteristic pattern of genetic aberrations in ovarian granulosa cell tumors. Mod Pathol ; 15(9):951-7

5. Gaffey MJ, Frierson HF Jr, Lezzoni JC, Mills SE, Clement PB, Shashi $V$ et al. Ovarian granulosa cell tumors with bizarre nuclei: an inmunohistochemical analysis with fluorescence in situ hybridization documenting trisomy 12 in the bizarre component. Mod Pathol; 9(3): 308-15;. Erratum Mod Pathol 9(7): 803;19

6. Tanyi J, Rigo J Jr, Csapo Z, Szentirmay Z Trisomy 12 in juvenile granulosa cell tumor of the ovary during pregnancy. A report of two cases. J Reprod Med ;44(9):826-32

7. Fletcher JA, Gibas Z, Donovan K, Perez-Atayde A, Genest D, Morton CC, Lage JM Ovarian granulosa-stromal cell tumors are characterized by trisomy 12. Am J Pathol; 138(3):515-20

8. Halperin D Fletcher, Visscher DW, Wallis T, Lawrence WD. Evaluation of chromosome 12 copy number in ovarian granulosa cell tumors using interphase cytogenetics. Int $J$ Gynecol Pathol; 14(4):319-23

9. Schwentner C, Oswald J, Rogatsch H, Mikuz G, Bartsch G, Radmayr C. Stromal testis tumors in infants. a report of two cases; 62(6):1121.

10. Taruscio $D^{1}$, Carcangiu ML, Ward DC. Detection of trisomy 12 on ovarian sex cord-stromal tumors by fluorescence In-situ hybridization. Diagn Mol Pathol; 2(2):94-8

11. Shashi V, Golden WL, von Kap-Herr C, Andersen WA, Gaffey MJ. Interphase fluorescence In-situ hybridization for trisomy 12 on archival ovarian sex cord-stromal tumors. Gynecol Oncol. 55(3 Pt 1):349-54.

12. Persons DL, Hartmann LC, Herath JF, Keeney GL, Jenkins RB.Fluorescence in situ hybridization analysis of trisomy 12 in ovarian tumors. Am J Clin Pathol; 102(6):775-9 
13. Lindgren V, Waggoner S, Rotmensh J Monosomy 22 in two ovarian granulosa cell tumor Cancer Genet Cytogenet 89(2):93-7

14. Leung WY, Schwartz PE, Heung-Tat NG, Yang-Feng TL . Trisomy 12 in benign fibroma and granulosa cell tumor of the ovary. Gynecol Oncol. 38(1):28-31

15. Van den Berghe I, Dal Cin P, De Groef K, Michielssen P, Van den Berghe $\mathrm{H}$ Monosomy 22 and trisomy 14 may be early events in the tumorigenesis of adult granulosa cell tumor. Cancer Genet Cytogenet; 1;112(1):46-8

16. Górski GK, McMorrow LE, Blumstein L, Faasse D, Donaldson $\mathrm{MH}$. Trisomy 14 in two cases of granulosa cell tumor of the ovary. Cancer Genet Cytogenet; 0(2):202-5

17. Caughron SK, Bridge JA, Bewtra CB, Hunter WJ, Nelson M, Soundararajan S, Silva E, Gatalica Z. Monosomy 22 as a diagnostic aid in a case of late recurrence of adult granulosa cell tumor of the ovary. Cancer Genet Cytogenet.;156(1):83-5

18. Namiq AL, Persons DL, Piehler J, Damjanov I.Monosomy 22 and trisomy 14 in a granulosa tumor metastatic to the lung 20 years after the removal of the primary tumor. Cancer Genet Cytogenet 1(2): 192-3

19. Bouffet E, Basset N, Chetai F, Dijoud P, Mollard P. BrunantMentigny M, David M. Juvenile granulosa cell tumor of the ovary in infants. Study of three cases and review of the literature. J Pediatr Surg; 22(6):762-765

20. Nistal M, Redondo E, Paniagua R Juvenile Granulosa Cell Tumor of the Testis. Arch Pathol Lab Med; 112(11): 1129-32

21. Alexiev BA, Alaish SM, Sun C. Testicular juvenile granulosa cell tumor in a newborn. Case report and review of the literature. Int. J Surg Path; 15:321-5

22. Couture J 2011', Bolduc S. A rare testicular solid mass in children: Juvenile granulosa cell tumour of testis. Can Urol Assoc J.;6(2):E101-3

23. Bulotta AL, Molinaro F, Angotti R, Ferrara F, Di Maggio G, Bindi $E$, Messina M. Juvenile granulosa cell tumor of the testis: prenatal diagnosis and prescrotal approach. Ital $\mathrm{J}$ Pediatr; 38:67

24. Kos M, Nogales FF, Kos M, Stipoljev F, Kunjko K.Congenital juvenile granulosa cell tumor of the testis in a fetus showing full 69,XXY triploidy; 13(2):219-21

25. Yu DC, Pathak B, Vargas SO, Javid PJ, Hisama FM, Wilson JM, Linden BC. Congenital intra-abdominal bilateral juvenile granulosa cell tumors of the testis associated with constitutional loss of material from chromosome 4. Pediatr Dev Pathol.; 14(3):224-7

26. Schofield DE, Fletcher JA. Trisomy 12 in pediatric granulosastromal cell tumors. Am J Pathol; 141(6): 1265-9

27. Young $\mathrm{RH}$, Lawrence DW, Scully RE Juvenile granulosa cell tumor-another neoplasm associated with abnormal chromosomes and ambiguous genitalia. A report of three cases Am J Surg Pathol; 9(10): 737-43

28. Raju U, Fine G, Warrier R, Kini R, Weiss L Congenital testicular juvenile granulose cell tumor in a neonate with $\mathrm{X} /$ XY mosaicism. Am J Surg Pathol (8): 577-83

29. Chan JK, Chan VS,.Mak KL. Congenital juvenile granulose cell tumour of the testis: report of a case showing extensive degenerative changes. Histopathology; 17(1):75-80

30. Tanaka Y, Sasaki Y, Tachibana K, Suwea S, Terashima K, Nakatani Y. Testicular juvenile granulosa cell tumor in an Infant with $X / X Y$ mosaicism clinically diagnosed as true hermaphroditism. Am. J. Surg. Pathol. 18(3): 316-22; 1994
31. Plesner KB, Jacobsen BB, Kock KE, Rix M, Rosthøj S. Granulosa cell tumors in children. Ugeskr Laeger. 2000 Jun 26;162(26):3731-3

32. Nitzsche K, Kamin G, Dittert DD, Bier A, Distler W. Fetal juvenile granulosa cell tumor with hermaphroditism verus prenatal diagnosis, management and outcome. Ultraschall Med.2009 Aug;.30 (4):404-7

33. Pascual J; McMann LP; Gallagher T; Pinsker JE. Ambiguous genitalia in a newborn with $45, \mathrm{X} / 46, \mathrm{X}$,idic(Y) ovotesticular disorder of sex development. Endocr Pract. 2009; 15(7):732-6

34. Forsberg LA, Rasi C, Malmqvist N, Davies H, Pasupulati S, Pakalapati $G$ y col Mosaic loss of chromosome $Y$ in peripheral blood is associated with shorter survival and higher risk of cancer. Nat Genet.;46(6):624-8

35. Reddy $\mathrm{KS}^{1}$, Sulcova V. Pathogenetics of $45, \mathrm{X} / 46, \mathrm{XY}$ gonadal mosaicism. Cytogenet Cell Genet. 1998;82(1-2):52-7

36. Nonomura K, Kakizaki H, Shibata T, Moriya K. Mixed gonadal disgenesis. Nihon Rinsho; 62(2):313-9

37. Motohashi $\mathrm{O}^{1}$, Shirane R, Ogawa A, Yoshimoto T.Tethered cord syndrome associated with male Turner's syndrome. Surg Neurol.; 40(1):57-60

38. Mansouri-Attia N, Tripurani SK, Gokul N, Piard H, Anderson ML, Eldin K, Pangas SA.TGF $\beta$ Signaling Promotes Juvenile Granulosa Cell Tumorigenesis by Suppressing Apoptosis. Mol Endocrinol. 2014; (11):1887-98

39. Boyer $A$, Paquet $M$, Laguê $M N$, Hermo $L$ Boerboom D Dysregulation of WNT/CTNNB1 and PI3K/AKT signaling in testicular stromal cells causes granulosa cell tumor of the testis. Carcinogenesis; 30(5): 869-878

40. Nomura $M^{1}$, Sakamoto $R$, Morinaga $H$, Wang L, Mukasa C, Takayanagi R.Activin stimulates CYP19A gene expression in human ovarian granulosa cell-like KGN cells via the Smad2 signaling pathway. Biochem Biophys Res Commun.;436(3):443-8

41. Zhang Y, Huang Q, Cheng JC, Nishi Y, Yanase T, Huang HF, Leung PC. Homeobox A7 increases cell proliferation by upregulation of epidermal growth factor receptor expression in human granulosa cells. Reprod Biol Endocrinol. 14;8:61

42. Shah, S. P., Kobel, M., Senz, J., Morin, R. D., Clark, B. A., Wiegand, K. C., Leung, G., Zayed, A., Mehl, E., Kalloger, S. E., Sun, M., Giuliany, R., and 29 others. Mutation of FOXL2 in granulosa-cell tumors of the ovary. New Eng. J. Med. 360: 2719-2729

43. Köbel M, Gilks CB, Huntsman DG. Adult-type granulosa cell tumors and FOXL2 mutation. Cancer Res; 15;69(24):9160-2

44. Jamieson S, Butzow R, Andersson N, Alexiadis M, UnkilaKallio L, Heikinheimo M, Fuller PJ, Anttonen M. The FOXL2 C134W mutation is characteristic of adult granulosa cell tumors of the ovary. Mod Pathol. 2010; (11):1477-85

45. Rosario R, Cohen PA, Shelling AN. The role of FOXL2 in the pathogenesis of adult ovarian granulosa cell tumours. Gynecol Oncol.;133(2):382-7

46. Kim MS, Granulosa cell tumour of ovary and other human cancers. J Pathol.;221(2):147-52

47. Kalfa N, Fellous M, Boizet-Bonhoure B, Patte C, Duvillard P, Pienkowski C, Jaubert F, Ecochard A, Sultan C Aberrant expression of ovary determining gene FOXL2 in the testis and juvenile granulosa cell tumor in children. J Urol; 180(4 Suppl):1810-3

E-mail: ppereda29@yahoo.com 